\title{
Research on counterexamples of improper integral
}

\author{
Yanying $\mathrm{Ma}^{1}$ Xue Wang ${ }^{2}$ and Yanli Liu ${ }^{3}$ \\ ${ }^{1}$ Jilin Engineering Normal University, Changchun 130052, China \\ ${ }^{2}$ Fuyang Teachers College, Fuyang 236041, China \\ ${ }^{3}$ No. 1 Senior High School of Jilin Chemical Industry Group of Jilin City, Jilin 132000, China
}

\begin{abstract}
The distinct differences between improper and definite integral is shown in this article by some counterexamples, by which we correct the students' misunderstanding and make the exclusive characters of improper integral clearer.
\end{abstract}

\section{Introduction}

Improper integral is an important knowledge point on integral after definite integral, which contains two typies, one with infinity integral limit is and the other with unbounded integrand. For the two are mutually transformable, we only research on infinite integral. Firstly, we think about whether the properties of integral could be passed on to or suit for improper integral, or which kind of properties could. According to the thinking way, we could enhance the students' understanding on the properties of improper integral by counterexamples.

\section{Properties different from definite integral}

1. When the improper integral $\int_{a}^{+\infty} f(x) d x$ is convergent, the integral $\int_{a}^{+\infty}|f(x)| d x$ may not be convergent too.

Example 1: The improper integral $\int_{1}^{+\infty} \frac{\sin x}{x} d x$ is convergent, but $\int_{1}^{+\infty}\left|\frac{\sin x}{x}\right| d x$ is divergent.

Solution: Let $F(a)=\int_{1}^{a} \sin x d x$, then $F(a)=\int_{1}^{a} \sin x d x$ is bounded, and $\lim _{x \rightarrow+\infty} \frac{1}{x}=0$, by Dirchlet test, we get $\int_{1}^{+\infty} \frac{\sin x}{x} d x$ is convergent, and $\left|\frac{\sin x}{x}\right| \geq \frac{\sin ^{2} x}{x}=\frac{1-\cos 2 x}{2 x}$. For $\int_{1}^{+\infty} \frac{\cos 2 x}{2 x} d x$ is convergent, but $\int_{1}^{+\infty} \frac{1}{2 x} d x$ is divergent, we get the conclusion that $\int_{1}^{+\infty}\left|\frac{\sin x}{x}\right| d x$ is divergent.

2. The infinite integral $\int_{a}^{+\infty} f(x) d x$ is convergent, but $\int_{a}^{+\infty} f^{2}(x) d x$ may not.

Example 2: Let $f(x)=\frac{\sin x}{\sqrt{x}}(1 \leq x<+\infty)$, then $\int_{1}^{+\infty} \frac{\sin x}{\sqrt{x}} d x$ is convergent, but $\int_{1}^{+\infty} \frac{\sin ^{2} x}{x} d x$ is divergent.

Solution: By Dirchlet test, the improper integral $\int_{1}^{+\infty} \frac{\sin x}{\sqrt{x}} d x$ is convergent, but the integral $\int_{1}^{+\infty} f^{2}(x) d x=\int_{1}^{+\infty} \frac{\sin ^{2} x}{x} d x=\int_{1}^{+\infty} \frac{1-\cos 2 x}{2 x} d x \quad$ is $\quad$ divergent obviously.

3[3]. If $\int_{a}^{+\infty} f(x) d x$ is absolutely convergent,the integral $\int_{a}^{+\infty} f^{2}(x) d x$ may not be convergent.

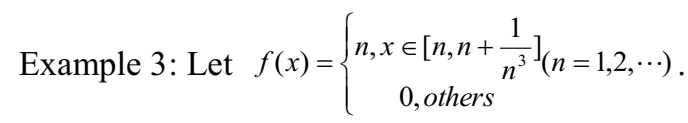

Solution: For $\quad \int_{1}^{+\infty}|f(x)| d x=\sum_{n=1}^{+\infty} \int_{n}^{n+1}|f(x)| d x=\sum_{n=1}^{+\infty} \frac{1}{n^{2}}=\frac{\pi^{2}}{6} \quad$ is convergent, the integral $\int_{1}^{+\infty} f(x) d x$ is absolutely convergent, but $\int_{1}^{+\infty} f^{2}(x) d x=\sum_{n=1}^{+\infty} \frac{1}{n}$ is divergent.

\section{Relation of integrand and the convergence of the integral}

Many students may think that, since improper integral and series have close connection, the general term tends to 0 when the series is convergent, then so does the convergent improper integral, namely the statement, that the integrand should approach 0 for a convergent improper integral, is not true.

4. If $\int_{a}^{+\infty} f(x) d x$ is convergent and $f(x)$ is non-negative on the interval $[a,+\infty)$, then $\lim _{x \rightarrow+\infty} f(x)=0$ may not be true.

Example 4 [2]: Set $f(\mathrm{x})=\left\{\begin{array}{c}\frac{a}{x}, x \text { is not natural } \\ 1, x \text { is natural }\end{array}\right.$.

Solution: For

\footnotetext{
a Corresponding author: guotianbao1999@126.com
} 


$$
\int_{1}^{+\infty} f(x) d x=\lim _{A \rightarrow+\infty} \int_{1}^{A} f(x) d x=\lim _{A \rightarrow+\infty}-\left.\frac{a}{x}\right|_{1} ^{A}=a,
$$

Then $\int_{1}^{+\infty} f(x) d x$ is convergent, but $\lim _{x \rightarrow+\infty} f(x)$ does not exist.

Note: The counterexample is constructed by the important property of definite integral, that is, when we change the function value on finite points, the integrability and integral value will not be affected.

5. If $\int_{a}^{+\infty} f(x) d x$ is convergent and $f(x)$ is continuous on $[a,+\infty)$, then $\lim _{x \rightarrow+\infty} f(x)=0$ may be untrue.

Example 5 [3]: Let $f(x)=\sin x^{2}(1 \leq x<+\infty)$.

Solution: The improper integral $\int_{1}^{+\infty} \sin x^{2} d x=\int_{1}^{+\infty} \frac{\sin t}{2 \sqrt{t}} d t$ is convergent, but $\lim _{x \rightarrow+\infty} \sin x^{2}$ does not exist, which indicts that the integrand may not tends to 0 even it is continuous.

Then we are curious about that in which cases, the integrand of a convergent integral will convergent to 0 ?

6 [2]. Set $f(x)$ is non-negative and continuous $[a,+\infty)$, the improper integral $\int_{a}^{+\infty} f(x) d x$ is convergent, then $\lim _{x \rightarrow+\infty} f(x)=0$.

Solution: The proposition is not true. Let $\{x\}=x-[x]$.

$I_{n}=\int_{\mathrm{n}}^{n+1} 4^{n}\{x\}(1-\{x\})^{n} d x=\int_{0}^{1} 4^{n} x^{n}(1-x)^{n} d x$

$=4^{\mathrm{n}} n \int_{0}^{1} x^{n}(1-x)^{n} d x=4^{n} B(n+1, n+1)=4^{n} \frac{(\mathrm{n} !)^{2}}{(2 n+1) !}$

$=\frac{(2 n) ! !}{(2 n+1) ! !}$.

Thus

$I_{\mathrm{n}}^{2}=\frac{2 \times 2 \times 4 \times 4 \times 6 \times \cdots \times(2 n-2) \times(2 n)}{1 \times 3^{2} \times 5^{2} \times \cdots \times(2 n-1)^{2}} \cdot \frac{2 n}{(2 n+1)^{2}}$

$<\frac{2}{2 n+1}$.

and

$$
I_{\mathrm{n}} \leq \sqrt{\frac{2}{2 n+1}}<\frac{1}{\sqrt{n}}
$$

Set $f(x)=4^{n^{3}}[\{x\}(1-\{x\})]^{n^{3}}, x \in[n, n+1), n=1,2, \cdots$.

Then $f(x)$ is $[1,+\infty)$ non-negative and continuous, and

$$
\int_{1}^{+\infty} f(x) d x=\sum_{n=1}^{\infty} I_{n}^{3}
$$

The expression (2) is convergent from (1), but $f\left(n+\frac{1}{2}\right)=1$.

Note: If the condition $f(x)$ is uniformly continuous on the interval $[a,+\infty)$, the integral $\int_{a}^{+\infty} f(x) d x$ is convergent, then the conclusion is hold.

7. Improper integral may not be divergent with unbounded integrand

Example 7. Set

$f(x)=(-1)^{n} \ln n, x \in\left[1+\frac{1}{2}+\cdots+\frac{1}{n-1}, 1+\frac{1}{2}+\cdots+\frac{1}{n}\right], n=1,2, \cdots$
Solution:

For $\lim _{n \rightarrow+\infty}(-1)^{n} \ln n$ does not exist, thus

$$
\int_{1}^{=\infty} f(x) d x=\sum_{n=1}^{+\infty} \int_{1+\frac{1}{2}+\cdots+\frac{1}{n-1}}^{1+\frac{1}{2}+\cdots+\frac{1}{n}}(-1)^{n} \ln n=\sum_{n=1}^{+\infty}(-1)^{n} \frac{\ln n}{n}=\sum_{n=1}^{+\infty} \int_{n}^{n+1} f(x) d x
$$

is convergent.

The above example shows that, the improper integral may convergent even the integrand is divergent.

8. When $\int_{a}^{+\infty} f(x) d x$ is convergent, the integrand may be unbounded.

Example 8: Let $f(x)$ is defined on $[a,+\infty)$ as follows:

$$
f(x)=\left\{\begin{array}{l}
n+1, x \in\left[n, n+\frac{1}{n(n+1)^{2}}\right], \quad n=1,2, \cdots \\
0, x \in\left(n+\frac{1}{n(n+1)^{2}}, n+1\right),
\end{array}\right.
$$

The function $f(x)$ is bounded, and $\int_{a}^{+\infty} f(x) d x=1$.

\section{Test the convergence of improper integrals}

9. If

$\lim _{x \rightarrow \infty} \frac{f(x)}{g(x)}=1$,

$\int_{a}^{+\infty} f(x) d x$ is convergent $\Leftrightarrow \int_{a}^{+\infty} g(x) d x$ is convergent.

)The statement is not true(

Example 9

Let $f(x)=\frac{\sin x}{\sqrt{x}}$

then $\int_{1}^{+\infty} f(x) d x=\int_{1}^{+\infty} \frac{\sin x}{\sqrt{x}} d x$ is continuous.

Set $g(x)=\left(1+\frac{\sin x}{\sqrt{x}}\right) \frac{\sin x}{\sqrt{x}}$,

then $\lim _{x \rightarrow \infty} \frac{f(x)}{g(x)}=1$,

but $\int_{1}^{+\infty} g(x) d x=\int_{1}^{+\infty} \frac{\sin x}{\sqrt{x}}\left(1+\frac{\sin x}{\sqrt{x}}\right) d x$

$$
=\int_{1}^{+\infty}\left(\frac{\sin x}{\sqrt{x}}+\frac{\sin ^{2} x}{x}\right) d x
$$

thus the improper integral $\int_{1}^{+\infty} g(x) d x$ is divergent.

Note: The comparison principle form is not the same as the text, if we add the condition that $f(x)$ and $g(x)$ are non-negative, the conclusion is hold. In order to avoid such misunderstanding, we should emphasize the principle is based on non-negative integrand in teaching.

10. Let $\int_{a}^{+\infty} f(x) d x$ is convergent, function $\varphi(x)$ is 
bounded, then the improper integral $\int_{a}^{+\infty} f(x) \varphi(x) d x$ may not be convergent.

Example 10.

Let $f(x)=\frac{\sin x}{x}$, then for $\forall A>1,\left|\int_{1}^{A} \sin x d x\right| \leq 2$, when $x \rightarrow+\infty$, we have $\frac{1}{x}$ is monotonic and tends to 0 .

The integral $\int_{1}^{+\infty} \frac{\sin x}{x} d x$ is convergent, $f(x)=\frac{\sin x}{x}$ has a discontinuous point at $x=0$, set the value at $x=0$, then $f(x)=\frac{\sin x}{x}$ can be regarded as continuous on $[0,1]$. Thus $\int_{0}^{+\infty} \frac{\sin x}{x} d x$ is convergent.

Set $\phi(x)=\sin x$ is bounded, $\int_{0}^{+\infty} \frac{\sin ^{2} x}{x} d x=\int_{0}^{+\infty} \frac{1-\cos 2 x}{2 x} d x$, for any $A>0$,we have

$\left|\int_{0}^{A} \cos 2 x d x\right|=\frac{1}{2}|\sin 2 A| \leq \frac{1}{2}$, and when $x \rightarrow+\infty, \frac{1}{2 x}$ tends to 0 monotonicly, by Dirichlet-test, we obtain $\int_{0}^{+\infty} \frac{\cos 2 x}{2 x} d x$ is convergent, but $\int_{0}^{+\infty} \frac{1}{2 x} d x$ is divergent, $\int_{0}^{+\infty} \frac{\sin ^{2} x}{x} d x$ is divergent

Note: If $\int_{a}^{+\infty} f(x) d x$ is absolutely convergent and $\varphi(x)$ is bounded on $[a,+\infty)$, so does $\int_{a}^{+\infty} f(x) \varphi(x) d x$.

\section{Conclusions}

This mathematical teaching method, constructing counterexamples, plays an important role in mathematical analysis. A good counterexample will make the students understand the definitions, theorems and principles more clearly.

\section{References}

1. J. Zhang, Journal of science of Teachers' college and University. 35, 23-25 (2015).

2. X. Wang, Y.L. Liu, X. Liu. Journal of Jilin Teachers Institute of Engineering and Technology. 31, 92-96 (2015)

3. S.W. Xiao, Studies in College Matheemaitics. 17, 123-126 (2014).

4. J.Y. Gao, J. Tu, Studies in College Mathematics. 18, (2015).

5. C.Y. Du, Research of Higher teaching. 26-28 (2008).

6. G.H. Deng, Hunan University of Techonlogy. 92-94 (2010).

7. Mathematical analysis. By East China Normal University, Fourth edition (volume 1). Higher education press. 282 (2011)

8. R.H. Liu, Y. Wang, Journal of Chifeng College (natural science edition). 14-15 (2009).

9. J.X. Chen, Higher education press. 363 (2011). 